\title{
Absorption Spectra of the Prototype Hot-Jupiters: determination of atmospheric constituents and structure
}

\author{
David K. Sing ${ }^{1}$, A. Lecavelier ${ }^{1}$, J.-M. Désert ${ }^{1}$, \\ A. Vidal-Madjar ${ }^{1}, \&$ G. Ballester ${ }^{2}$ \\ ${ }^{1}$ Institut d'Astrophysique de Paris, CNRS; Université Pierre et Marie Curie, 98 bis bv Arago, \\ F-75014 Paris, France; sing@iap.fr \\ ${ }^{2}$ Lunar and Planetary Laboratory, University of Arizona, Sonett Space Science Building,
} Tucson, AZ 85721-0063, USA

\begin{abstract}
The two prototype hot-Jupiter exoplanets HD209458b and HD189733b are currently offering an unprecedented view of their atmospheres. As discussed here, primary transit transmission spectra provide the opportunity to identify specific atomic and molecular species, determine their abundances, and recover temperature-pressure-altitude information. We present a reanalysis of existing HST/STIS data on HD209458b, providing a complete optical transmission spectrum. Analysis of this spectrum have revealed: (1) the planetary abundance of sodium which is $\sim 2 \mathrm{X}$ solar (2) a depletion of sodium at high altitudes due to condensation or ionization (3) Rayleigh scattering by $\mathrm{H}_{2}$ (3) a high temperature at pressures of 10's mbar consistent with the dayside inversion (4) a separate high-altitude hot temperature from the planet's thermosphere and (5) likely absorption by TiO/VO. While HD209458b and HD189733b are currently the best candidates for these studies, another $\sim 10$ exoplanets are good targets with today's instruments for future transmission-based atmospheric detections.
\end{abstract}

\section{Which Exoplanets Have Detectable Atmospheres During Transit?}

Primary transit transmission spectra probes absorption depth, which is a measure of planetary altitude, $z$, which has a wavelength dependance. Detecting atmospheric signatures become easier with: (1) brighter parent stars, as photometry becomes easier (2) deeper transit depths, which provides larger contrast/signal and (3) a larger atmosphere, resulting from lower surface gravities or higher temperatures providing large scale heights, $H=k T / \mu g$. The measured altitude depends upon the atmospheric composition as well as its physical state and can be approximated by,

$$
z(\lambda)=H \ln \left(\frac{\xi_{a b s} P_{z=0} \sigma_{a b s}}{\tau_{e q} \mu g} \sqrt{\frac{2 \pi R_{p}}{H}}\right)
$$

(see Lecavelier et al. 2008a) where $\xi_{a b s}$ is the abundance of absorbing species, $P_{z=0}$ is the zero altitude pressure, $R_{p}$ is the planetary radius, $\mu$ is the mean mass of the atmospheric particles, $\sigma$ is the cross section and $\tau_{e q}$ is the equivalent optical depth. A transit atmospheric transmission signal is dominated by the absorption directly at the planetary terminator over one scale height, as the line of sight column density drops rapidly both horizontally and vertically, mitigating the effects of both vertical and horizontal temperature gradients.

With current instruments, only a sub-set of the known transiting planets will have detectable atmospheres, though the list is growing rapidly as detection surveys are becoming ever more efficient. A plot of the transit signal of an atmospheric scale height vs. 


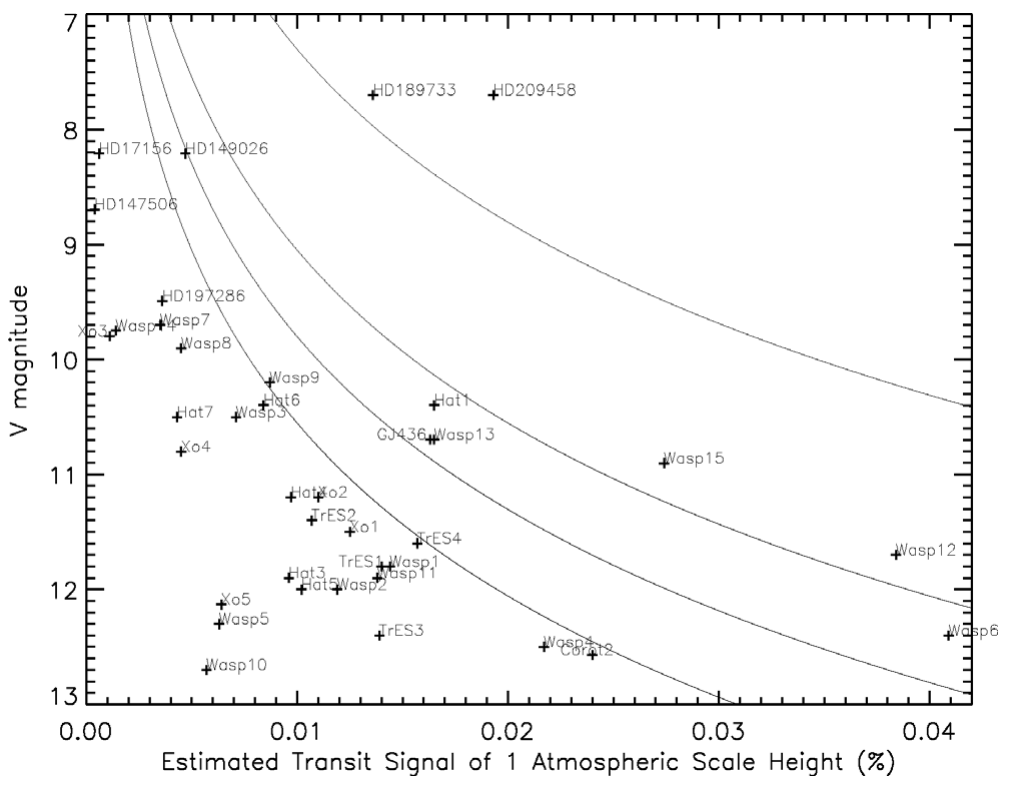

Figure 1. Atmospheric transit signals vs. stellar V magnitude. The top curve indicates the corresponding precision achieved per resolution element when integrating for 90 minutes $(\sim 1$ transit) with an HST/STIS low-resolution grating. The bottom three curves are precision estimates when increasing the collected photons by a factor of 5,10 , and 20 respectively. Planetary values obtained from Pont (2008) http://www.inscience.ch/transits/, Schneider (2008) http://exoplanet.eu/, and references therein.

magnitude, seen in Fig. 1, provides a good indication of which targets are best suited for transmission spectral observations. For the figure, $H$ was estimated using the exoplanet's equilibrium temperature, $\mu$ to be 2.3 times the mass of the proton, and the signal calculated from area of the obscuring atmospheric ring compared to area of the stellar disk. The actual atmospheric transit signals can naturally extend over many scale heights, depending on the atmospheric constituents and their opacities. HD209458b \& HD189733b are the best candidates, and HST can reach the relevant precisions observing just one transit at the native spectral resolution of STIS. Reaching fainter targets requires collecting more photons, which can be achieved by integrating multiple transits, using larger telescopes, using more efficient instruments, or binning in wavelength (at the expense of resolution).

\section{Decoding Transmission Spectra: how can $T-P-z$ be determined?}

Good spectral resolution, high $\mathrm{S} / \mathrm{N}$, and large wavelength coverage makes it possible to disentangle the absorption from multiple species. With absorbing species identified, knowledge of the wavelength dependent cross section provides a determination of the local temperature at altitude $z$, as $H$ can be determined. From Lecavelier et al. (2008a),

$$
T=\frac{\mu g}{k}\left(\frac{d l n \sigma}{d \lambda}\right)^{-1} \frac{d z(\lambda)}{d \lambda} .
$$

In the case of $\mathrm{H}_{2}$ Rayleigh scattering, this provides a determination of the temperature and the pressure, as the abundance of hydrogen in hot-Jupiters can be assumed to be unity (Lecavelier et al. 2008b), which sets the pressure scale, $P_{z=0}$, for all other 


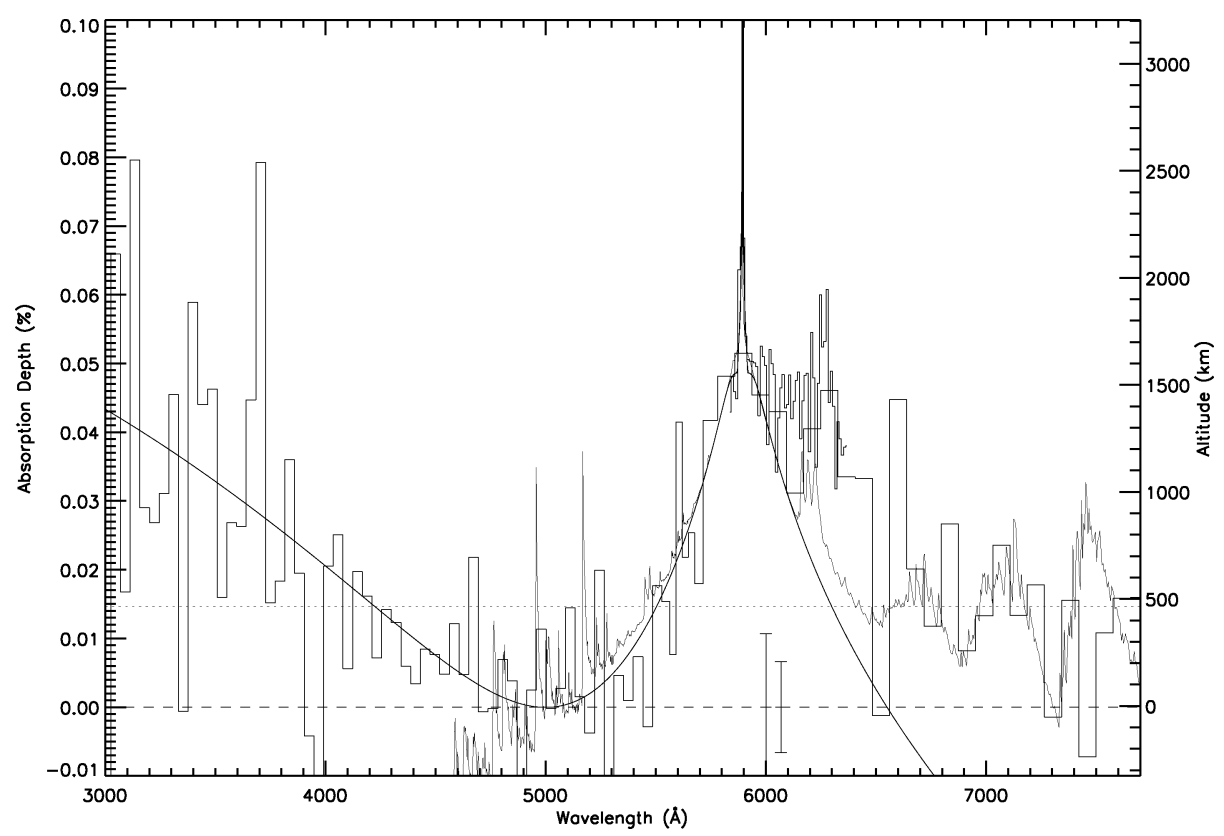

Figure 2. Transit Na D absorption from Sing et al. (2008a,b). Plotted is the planetary ab-

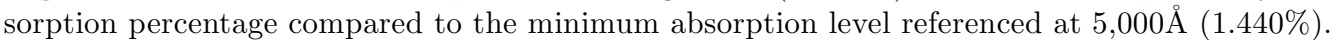
In black is the low resolution data binned over 16 pixels and over-plotted in dark grey is the medium resolution data binned over 18 pixels $(10 \AA)$. Representative error bars for the binned data are also plotted (also see Fig. 10 \& 11 of Sing et al. 2008a,b) as well as the mean planetary radius from Knutson et al. 2007 (dotted line). Also plotted (dark) is our transit absorption model which contains opacity from atomic Na, Rayleigh scattering, and contains upper-atmospheric $\mathrm{Na}$ depletion. The model (light) includes $\mathrm{Na}, \mathrm{TiO}$, and VO illustrating the wavelengths where those features are prominent. Higher absorption values indicate higher atmospheric altitudes, with the right $y$-axis labeled as the altitude above our minimum absorption depth. The narrow $\mathrm{Na} \mathrm{D} 1$ and D2 cores extend above a wide plateau of $\mathrm{Na}$ absorption indicating a low amount of $\mathrm{Na}$ is present in the middle atmosphere (above $\sim 1500 \mathrm{~km}$ ) compared to the lower.

wavelengths. With the pressure at an altitude known, the abundances of other observed species can then be determined at that altitude, as was done for sodium in Sing et al. (2008a) for HD209458b.

\section{HD209458b}

The STIS transit spectrum details of HD209458b presented here is found in Sing et al. (2008a,b), Lecavelier et al. (2008), and Désert et al. (2008) and builds off of the previous work of Ballester et al. (2007), Knutson et al. (2007), and Charbonneau et al. (2002). In our re-analysis we find that the spectrum shows:

(1) Sodium: The original Na detection by Charbonneau et al. (2002) is placed in context with the rest of the spectrum and the small signal is not due to opaque clouds, but rather from either $\mathrm{Na}$ condensation or ionization. We measure the Na abundance in the lower atmosphere to be $2 \mathrm{X}$ solar. At high altitudes and pressures below a few mbar, the abundance of $\mathrm{Na}$ drops suddenly to values of 0.2 solar or lower (see Sing et al. 2008a,b).

(2) Thermosphere detection: The core of the Na lines persists to altitudes high enough to reach the thermosphere, as indicated by the hot high altitude temperatures we find. 
(3) Rayleigh scattering: The absorption blue-ward of $5000 \AA$ is likely due to Rayleigh scattering by $\mathrm{H}_{2}$. In this case, it is possible to determine absolute pressures and temperatures from the spectrum (Lecavelier et al. 2008).

(4) $\mathrm{TiO} / \mathrm{VO}$ : Absorption by $\mathrm{TiO} / \mathrm{VO}$ fits the remainder of the transmission spectrum well and is consistent with the temperatures measured from Rayleigh scattering (Désert et al. 2008), though the $\mathrm{S} / \mathrm{N}$ is not sufficient to observe specific molecular band-heads.

The sodium core observed in the HST spectrum has been confirmed from ground based measurements by Snellen et al. (2008) with the results matching very well. The groundbased and HST sodium data together strengthens the need for a high-altitude hot temperature and the detection of the thermosphere.

\section{Conclusions}

With regular space-based and ground-based detections, transmission spectroscopy is entering the era of comparative exoplanetology which will give invaluable information at a specific planetary location, namely the terminator. Ground-based detections are complimentary to space-based data, as very high resolution spectra can be obtained and specific strong narrow absorbing species like Na can be detected, though the information of transit depth is lost in these measurements (Redfield et al. 2007; Snellen et al. 2008). Space-based transit spectra can retain the transit depth information making it possible to compare absorption depths over the entire observable wavelength range. Secondary transit measurements, as those done by Spitzer, also offer completely complementary information to that of transmission spectra. Information from all these observing methods will be needed at as many wavelengths as possible, at as high a $\mathrm{S} / \mathrm{N}$ as possible, and at as high a resolution as possible, for as many planets as possible, in order for the community to have a robust and comprehensive understanding of the relevant physical processes in these exoplanets.

\section{References}

Ballester, G. E., Sing, D. K., \& Herbert, F. 2007, Nature, 445, 511-514

Charbonneau, D., Brown, T. M., Noyes, R. W., \& Gilliland, R. L. 2002, ApJ, 568, 377-384

Désert, J.-M., Vidal-Madjar, A., Lecavelier des Etangs, A., Sing, D. K., Ehrenreich, D., Hebrard, G., \& Ferlet, R. 2008 A\&A, submitted

Knutson, H. A., Charbonneau, D., Noyes, R. W., Brown, T. M., \& Gilliland, R. L. 2007, ApJ, $655,564-575$

Lecavelier des Etangs, A., Pont, F., A., Vidal-Madjar, A., \& Sing, D. K. 2008a, A\&A, 481, 83

Lecavelier des Etangs, A., Vidal-Madjar, A., \& Désert, J. M., \& Sing, D. K. 2008b, A\&A, accepted, arXiv:0805.0595

Redfield, S., Endl, M., Cochran, W., \& Koesterke, L. 2007, ApJ, 673, 87-90

Sing, D. K., Vidal-Madjar, A., Désert, J.-M., Lecavelier des Etangs, A., \& Ballester, G. 2008, ApJ, accepted, arXiv:0802.3864

Sing, D. K., Vidal-Madjar, A., Lecavelier des Etangs, A., Désert, Ballester, \& G. Ehrenreich, D. 2008, ApJ, accepted, arXiv:0803.1054

Snellen, I. A. G., Albrecht S., De Mooij, E. J. W., \& Le Poole, R. S. 2008, A\&A, accepted, arXiv:0805.0789 